\title{
Unfolding and effective bandstructure calculations as discrete real- and reciprocal-space operations
}

\author{
Timothy B. Boykin ${ }^{\mathrm{a}, *}$, Arvind Ajoy ${ }^{\mathrm{c}}$, Hesameddin Ilatikhameneh ${ }^{\mathrm{b}}$, Michael Povolotskyi ${ }^{\mathrm{b}}$, and \\ Gerhard Klimeck $^{\mathrm{b}}$ \\ ${ }^{a}$ Department of Electrical and Computer Engineering, The University of Alabama in Huntsville, \\ Huntsville, Alabama 35899 USA \\ ${ }^{\mathrm{b}}$ Network for Computational Nanotechnology, School of Electrical and Computer Engineering, \\ Purdue University, West Lafayette, Indiana 47907 USA \\ ${ }^{\mathrm{c}}$ School of Electrical and Computer Engineering, Cornell University, Ithaca, New York 14853 \\ $U S A$
}

\begin{abstract}
In recent years, alloy electronic structure calculations based on supercell Brillouin zone unfolding have become popular. There are a number of formulations of the method which on the surface might appear different. Here we show that a discrete real-space description, based on discrete Fourier transforms, is fully general. Furthermore, such an approach can more easily show the effects of alloy scattering. We present such a method for treating the random alloy problem. This treatment features straightforward mathematics and a transparent physical interpretation of the calculated effective (i.e., approximate) energy bands.
\end{abstract}

Keywords: unfolding; supercell; alloy

*Corresponding author: boykin@ece.uah.edu 


\section{Introduction}

Approximate concepts in physics are often the most difficult to articulate and quantify, but quantification is essential for it establishes the range of validity of the approximation. The alloy concept is one which is particularly easy to explain in qualitative terms but which can be problematic to quantify. Semiconductor alloys are of particular technological interest, being found in lasers and transistors incorporated into nearly all consumer and industrial electronic products. The dispersions of semiconductor random-alloy structures (e.g., superlattices and resonant-tunneling diodes) have in fact been measured, by angle-resolved photoemission spectroscopy[1] and resonant magnetotuneling spectroscopy[2]. Because alloy-based devices are so small (many are nanometer scale) quantitative descriptions of semiconductor alloys are essential.

A very attractive and useful approach for alloy problems, both bulk and nanostructure, is supercell-based Brillouin zone unfolding. The idea behind an alloy effective bandstructure calculation based on supercell unfolding is to calculate approximate primitive cell band energies using the exact supercell eigenstates. Because the alloy only has approximate translational symmetry on the primitive cell level, these energy expectation values are not the same as the supercell energies, and there are no primitive cell periodic stationary states. The approximate primitive cell energy bands thus have finite energy uncertainties, due to the alloy disorder. Alloy scattering is therefore automatically included in the calculation and leads transparently to the energy uncertainties.

The process of converting the many supercell bands in a small Brillouin zone to the few primitive cell bands in a large Brillouin zone is called "unfolding," because pictorially it appears like unfolding a map. Random alloy models based on supercell unfolding have clear physics, 
with the underlying electronic structure calculation taking the following form: (1) Randomly populate a supercell (SC) with primitive cells (PCs) with the constituent atoms according to their respective mole fractions and at positions within the cells which minimize the energy; (2) Impose periodicity over the SC to obtain its energy bands; (3) Project out of the eigenstates the contributions from the allowed PC wavevectors (which are SC reciprocal lattice vectors). Step (3), commonly referred to as "unfolding," at its core really amounts to a discrete Fourier transform relationship, and by tracking the contributions of the various SC eigenstates to PC states of a given wavevector, one can determine approximate PC band energies and their associated uncertainties in the usual manner. The above procedure can also be used to find the approximate phonon bands (vibrational modes) of an alloy.

There are a number of ways to describe the unfolding process[3-16], and it is well-suited to both localized orbital (tight-binding) and extended (plane-wave) bases. However, when any of these methods is implemented on a computer there is an explicit discretization of either real or reciprocal space, and the discretization of one automatically implies discretization of the other; we illustrate this point in Appendix A. Here we use the tight-binding model to illustrate the method without loss of generality, because any finite set of wavevectors automatically implies the existence of a discrete real-space grid. Our presentation concentrates on the ideas of basis change and the use of Fourier methods to accomplish the unfolding.

\section{Unfolding}

\subsection{General considerations}

Before developing the unfolding method we address two closely related issues: (1) the generality of a localized (tight-binding) basis; and (2) the use of a periodic basis to represent an 
operator which is not periodic. Both issues are discussed extensively in the Appendix; here we examine the relevance for unfolding.

Any useful unfolding calculation must be performed on a computer. As such, the number of basis functions employed must be finite. Because we will populate a SC with possibly different PCs and impose periodic boundary conditions over the SC, the interest for unfolding is in periodic functions. In Appendix A we show that when a finite number of harmonics is retained in a Fourier series, the continuous periodic function can only be exactly represented on a finite grid of points, and that this grid is defined by (i.e., is reciprocal to) the retained harmonics. Thus, any calculation, even one based on plane waves, can only exactly represent the function on the finite grid of real-space points. This grid in turn defines a real-space localized basis. (In the case of plane waves it is Kronecker delta functions.)

The second question concerns the use of a periodic basis for non-PC-periodic wavefunctions. Mathematically, this is exactly analogous to taking the discrete Fourier transform of an aperiodic discrete-time (or discrete-space) function[17]. Indeed, imposing periodicity on the SC corresponds to Nyquist-rate sampling with no zero padding in discrete signal processing. (Note that a plane-wave calculation uses PC-periodic plane waves to represent non-PC-periodic wavefunctions and Hamiltonian operators.) We discuss this issue extensively following the unfolding method. The disorder is confined to the Hamiltonian operator, not the basis, and appears in the Hamiltonian matrix elements. There we also show that in the PC-periodic basis the PC-periodic part of the Hamiltonian automatically appears as the diagonal blocks of its matrix while the disorder part automatically appears as the off-diagonal blocks.

The unfolding procedure is valid regardless of the degree of disorder in the SC - one can always Fourier decompose a well-behaved non-periodic discrete function. As a purely 
mathematical operation, one can discrete Fourier transform a scalar function of three spatial variables onto an any three-dimensional $\mathbf{k}$-space grid of SC reciprocal lattice vectors. Minimal requirements for extracting useful physical information are that that dividing the SC into identical-size PCs makes sense and using the $\mathbf{k}$-space grid for these PCs. The extent to which the resulting Fourier spectrum conveys useful physics depends on the degree of disorder in the SC. If the SC is perfectly periodic, the unfolding process yields exact PC bands, and even if the SC has large strains, one can always define PCs based on the average SC dimension to generate the PC wavevectors for unfolding. If the strains are so great that one cannot well represent the SC using average-sized PCs, the unfolded approximate PC bands will have little physical meaning: The SC essentially has little PC-level translational symmetry.

The degree of SC disorder appears in both the projected PC-periodic bands as well as the SC Hamiltonian expressed in a PC-periodic basis. A SC with little disorder has a Hamiltonian matrix in the PC-periodic basis with dominant diagonal blocks and small off-diagonal blocks. In this case the energy uncertainties will be small compared to the separations between the bands. When the SC disorder is large, the off-diagonal blocks are likewise significant, and the eigenstates can no longer be considered to be eigenstates of a PC-periodic Hamiltonian. The energy uncertainties will be large, indicating strong coupling between the bands.

\subsection{Unfolding as a Discrete Fourier Transform}

We consider a very large solid, consisting of $N_{S}$ SCs, over which we enforce Born-von Karman boundary conditions. The SC eigenstates are therefore Bloch states of one of $N_{S} \mathrm{SC}$ wavevectors $\mathbf{K}_{s}$ belonging to the SC first Brillouin zone. The exact number of SCs and the quantization of the $\mathbf{K}_{s}$ are not important here. As discussed in Appendix A the choice of a grid 
for the $\mathbf{K}_{s}$ will automatically determine these properties, so from here we drop its index. Each of the identical SCs contains $N_{C}$ PCs, which may differ. The PCs themselves are arranged in a regular array, but the composition and exact positions of the atom(s) within may vary from PC to PC. Specifically, we take each SC to have $N_{i}, i=1,2,3$, PCs along each of the PC direct lattice vectors, $\mathbf{a}_{i}, i=1,2,3$, such that $N_{C}=N_{1} N_{2} N_{3}$. The same basis is used for all PCs, with $N_{O}$ basis functions per PC, indexed by $\omega$. (This is a compound index covering both atom and orbital type, $s, p_{x}$, etc.) The SCs are located at positions $\mathbf{R}_{j}$ and within a SC the PCs are located at positions $\boldsymbol{\rho}_{l}$ relative to the SC origin; the $\boldsymbol{\rho}_{l}$ are the same for all SCs. At each $\mathbf{K}$ there are $N_{O} N_{C}$ SC eigenstates; that of energy $E_{p}$ is written as:

$$
\left|\Psi_{p}(\mathbf{K})\right\rangle=\sum_{j=1}^{N_{S}} \sum_{\omega=1}^{N_{o}} \sum_{l=1}^{N_{C}} \frac{e^{i \mathbf{K} \cdot \mathbf{R}_{j}}}{\sqrt{N_{S}}} \beta_{l, p}^{(\omega)}(\mathbf{K})\left|\omega ; \mathbf{R}_{j}+\boldsymbol{\rho}_{l}\right\rangle
$$

On the other hand, these same SC eigenstates can be expanded in terms of PC-periodic Bloch states. At this point all that is important is that there is a set of PC-periodic, orthonormal Bloch band states[4,8]. Using this basis is exactly analogous to obtaining the spectrum of an aperiodic function or signal. There are $N_{C}$ PC wavevectors $\mathbf{k}_{n}=\mathbf{K}+\mathbf{G}_{n}, n=1, \ldots, N_{C}$ within the PC first Brillouin zone and $N_{o}$ orthonormal Bloch states (bands) at each $\mathbf{k}_{n}$, indexed by $\eta$ :

$$
\left|\psi_{\eta}\left(\mathbf{K}+\mathbf{G}_{n}\right)\right\rangle=\sum_{j=1}^{N_{S}} \sum_{\omega=1}^{N_{o}} \sum_{l=1}^{N_{C}} \frac{e^{i \mathbf{K} \cdot \mathbf{R}_{j}}}{\sqrt{N_{S} N_{C}}} b_{\eta}^{(\omega)}\left(\mathbf{K}+\mathbf{G}_{n}\right) e^{i\left(\mathbf{K}+\mathbf{G}_{n}\right) \cdot \mathbf{p}_{l}}\left|\omega ; \mathbf{R}_{j}+\mathbf{\rho}_{l}\right\rangle
$$

These bands are not explicitly defined at this stage. It is sufficient that such a basis does exist: The $\left|\psi_{\eta}\left(\mathbf{K}+\mathbf{G}_{n}\right)\right\rangle$ and the $\left|\omega ; \mathbf{R}_{j}+\boldsymbol{\rho}_{l}\right\rangle$ do, after all, span the same space. If the SC is perfect (all PCs identical) the unfolding process will even recover the $b_{\eta}^{(\omega)}$ for a non-degenerate band[3]. 
The $\mathbf{G}_{n}$ are the $N_{C}$ SC reciprocal lattice vectors falling within the PC first Brillouin zone and are defined in terms of the PC reciprocal lattice vectors $\mathbf{b}_{i}, i=1,2,3$ as[3,4]:

$$
\mathbf{G}_{n}=\sum_{j=1}^{3} \frac{n_{j}}{N_{j}} \mathbf{b}_{j}, n_{j}=\left\{\begin{array}{rr}
-\frac{\left(N_{j}-2\right)}{2}, \ldots, 0, \ldots \frac{N_{j}}{2}, & N_{j} \text { even } \\
-\frac{\left(N_{j}-1\right)}{2}, \ldots, 0, \ldots \frac{\left(N_{j}-1\right)}{2}, & N_{j} \text { odd }
\end{array}\right.
$$

In eq. (3), $n$ is a compound index representing the $N_{C}$ trios $\left(n_{1}, n_{2}, n_{3}\right)$. If any of the $\mathbf{k}_{n}=\mathbf{K}+\mathbf{G}_{n}$ falls outside the PC first Brillouin zone due to non-zero $\mathbf{K}$, it is shifted back by adding the appropriate PC reciprocal lattice vector. The unfolding method can even recover PC bands in the case where the "PCs" are not truly primitive (e.g., the 4-PC cube for FCC), but determining the allowed $\mathbf{k}_{n}=\mathbf{K}+\mathbf{G}_{n}$ is more involved[18-19]. Figure 1 shows the direct and reciprocal lattices (PC and SC) for a two-dimensional square $2 \times 2 \mathrm{SC}$.

Thus there are $N_{O} N_{C} \mathrm{SC}$ eigenstates, eq. (1), and $N_{O}$ PC energy bands at each of the $N_{C}$ allowed PC wavevectors, eq. (2). Each SC eigenstate is generally a linear combination of all $N_{O} N_{C}$ PC-band states, since the alloy disorder couples states of different PC wavevector:

$$
\left|\Psi_{p}(\mathbf{K})\right\rangle=\sum_{\eta=1}^{N_{O}} \sum_{n=1}^{N_{C}} a_{p ; \eta, n}\left|\psi_{\eta}\left(\mathbf{K}+\mathbf{G}_{n}\right)\right\rangle
$$

Now taking the inner product of eq. (4) with one of the localized basis states $\left\langle\omega ; \mathbf{R}_{j}+\boldsymbol{\rho}_{l}\right|$ and rearranging leads to one of $N_{C}$ equations[4]:

$$
e^{-i \mathbf{K} \boldsymbol{p}_{l}} \beta_{l, p}^{(\omega)}(\mathbf{K})=\frac{1}{\sqrt{N_{C}}} \sum_{n=1}^{N_{C}} \sum_{\eta=1}^{N_{O}} a_{p ; \eta, n} b_{\eta}^{(\omega)}\left(\mathbf{K}+\mathbf{G}_{n}\right) e^{i \mathbf{G}_{n}\left\lceil\boldsymbol{p}_{l}\right.}
$$

In matrix form, these equations (one per PC) read:

$$
\mathbf{B}_{p}^{(\omega)}(\mathbf{K})=\underline{\mathbf{U}} \cdot \mathbf{C}_{p}^{(\omega)}(\mathbf{K})
$$


where the components of the $N_{C}$-component vectors and $N_{C} \times N_{C}$ unitary matrix are:

$$
\left[\mathbf{B}_{p}^{(\omega)}(\mathbf{K})\right]_{m}=e^{-i \mathbf{K} \cdot \boldsymbol{\rho}_{m}} \beta_{m, p}^{(\omega)}(\mathbf{K}), \quad\left[\mathbf{C}_{p}^{(\omega)}(\mathbf{K})\right]_{m}=\sum_{\eta=1}^{N_{O}} a_{p ; \eta, m} b_{\eta}^{(\omega)}\left(\mathbf{K}+\mathbf{G}_{m}\right), \quad[\underline{\mathbf{U}}]_{m, n}=\frac{1}{\sqrt{N_{C}}} e^{i \mathbf{\rho}_{m} \cdot \mathbf{G}_{n}}
$$

Because the matrix is unitary the solution of eq. (6) is easily obtained:

$$
\left[\mathbf{C}_{p}^{(\omega)}(\mathbf{K})\right]_{m}=\frac{1}{\sqrt{N_{C}}} \sum_{j=1}^{N_{C}} e^{-i\left(\mathbf{K}+\mathbf{G}_{m}\right) \cdot \mathbf{\rho}_{j}} \beta_{j, p}^{(\omega)}(\mathbf{K})
$$

A brief examination shows that the vector $\mathbf{C}_{p}^{(\omega)}(\mathbf{K})$ is just the discrete Fourier transform of the vector $\mathbf{B}_{p}^{(\omega)}(\mathbf{K})$ [17]. Thus unfolding reduces to a discrete Fourier transform operation.

We have now obtained the spectrum of the $p$-th $S C$ eigenstate. From it, we find the probability that this SC eigenstate has PC-periodic components of wavevector $\mathbf{k}_{n}=\mathbf{K}+\mathbf{G}_{n}$ [4]:

$$
\mathcal{P}_{p, n}=\sum_{\omega=1}^{N_{O}}\left|\left[\mathbf{C}_{p}^{(\omega)}(\mathbf{K})\right]_{n}\right|^{2}=\sum_{\eta=1}^{N_{O}}\left|a_{p ; \eta, n}\right|^{2}
$$

In the last step of eq. (9) we used the orthonormality of the PC-periodic Bloch bands, eq. (2). The interpretation of the $\mathscr{P}_{p, n}$ as probabilities follows from eq. (4):

$$
\left|a_{p ; \eta, n}\right|^{2}=\left\langle\psi_{\eta}\left(\mathbf{K}+\mathbf{G}_{n}\right) \mid \Psi_{p}(\mathbf{K})\right\rangle\left\langle\Psi_{p}(\mathbf{K}) \mid \psi_{\eta}\left(\mathbf{K}+\mathbf{G}_{n}\right)\right\rangle
$$

and the closure relation in the PC-periodic basis,

$$
\begin{gathered}
\hat{\mathbf{1}}_{\mathbf{K}}=\sum_{n=1}^{N_{C}} \sum_{\eta=1}^{N_{O}}\left|\psi_{\eta}\left(\mathbf{K}+\mathbf{G}_{n}\right)\right\rangle\left\langle\psi_{\eta}\left(\mathbf{K}+\mathbf{G}_{n}\right)\right| \\
\sum_{n=1}^{N_{C}} \mathscr{P}_{p, n}=\sum_{n=1}^{N_{C}} \sum_{\eta=1}^{N_{O}}\left|a_{p ; \eta, n}\right|^{2}=\left\langle\Psi_{p}(\mathbf{K}) \mid \Psi_{p}(\mathbf{K})\right\rangle=1
\end{gathered}
$$

Thus eq. (12) shows that $\mathscr{P}_{p, n}$ is the contribution to the $p$-th SC state from all PC states of $\mathbf{k}_{n}=\mathbf{K}+\mathbf{G}_{n}$. There is another sum rule, essential for determining the effective alloy bands, which follows from eqs. (9) and (10) along with the closure relation in the SC-periodic basis: 


$$
\begin{gathered}
\hat{\mathbf{1}}_{\mathbf{K}}=\sum_{p=1}^{N_{C} N_{O}}\left|\Psi_{p}(\mathbf{K})\right\rangle\left\langle\Psi_{p}(\mathbf{K})\right| \\
\sum_{p=1}^{N_{c} N_{O}} \mathscr{P}_{p, n}=\sum_{p=1}^{N_{C} N_{o}} \sum_{\eta=1}^{N_{O}}\left|a_{p ; \eta, n}\right|^{2}=\sum_{\eta=1}^{N_{O}}\left\langle\psi_{\eta}\left(\mathbf{K}+\mathbf{G}_{n}\right) \mid \psi_{\eta}\left(\mathbf{K}+\mathbf{G}_{n}\right)\right\rangle=N_{O}
\end{gathered}
$$

That is, summing the probabilities for PC-wavevector $\mathbf{k}_{n}=\mathbf{K}+\mathbf{G}_{n}$ over all $N_{O} N_{C}$ SC eigenstates gives the number of PC-basis bands. This sum rule suggests a way to determine effective PC bands: Plot the cumulative probability for the PC-wavevector $\mathbf{k}_{n}=\mathbf{K}+\mathbf{G}_{n}$ as a function of SC energies $E_{p}$ and look for steps. A step of size 1 indicates a non-degenerate band has been passed with increasing energy; a step of size 2 indicates that a doubly-degenerate (or nearly degenerate) pair of PC bands has been passed[4].

When a PC-periodic band description is appropriate there will be fairly sharp steps in the cumulative probability, and there will be zero or only negligible probability contributions from SC states at energies in the gaps between band edges. By including the SC states which contribute to a cumulative probability step of size 1 we can calculate effective PC-periodic band energies[4]. That these average energies are true expectation values follows from the Hamiltonian in the SC eigenstate basis and eq. (4):

$$
\begin{gathered}
\hat{H}=\sum_{p=1}^{N_{C} N_{O}}\left|\Psi_{p}(\mathbf{K})\right\rangle E_{p}\left\langle\Psi_{p}(\mathbf{K})\right| \\
\bar{E}_{\eta}\left(\mathbf{K}+\mathbf{G}_{n}\right)=\left\langle\psi_{\eta}\left(\mathbf{K}+\mathbf{G}_{n}\right)|\hat{H}| \psi_{\eta}\left(\mathbf{K}+\mathbf{G}_{n}\right)\right\rangle=\sum_{p=1}^{N_{c} N_{o}} E_{p}\left|a_{p ; \eta, n}\right|^{2}
\end{gathered}
$$

Averaging the probability-weighted energies of SC states contributing to a step of size 1, we determine which $a_{p ; \eta, n}$ are nonzero: Probabilities for SC states above or below the step are not 
included in the sum, so their $a_{p ; \eta, n}$ are zero for this PC-periodic band. This analysis is easily extended to the case of degenerate or nearly degenerate bands[4].

Because the somewhat differing physics of unfolding problems, such as the electrical and vibrational structure of a random alloy, the exact criteria for band determination vary. Electronic structure problems such as the AlGaAs alloy[4] tend to have sharper steps in the cumulative probability for single band-edges. The vibrational problem for InGaAs alloys[6] involves both significant strain (affecting the neighboring atom couplings) and mass differences between the two possible cations (affecting all matrix elements), which help lead to smoother cumulative probability steps. Because these effective bands are not eigenstates of the SC Hamiltonian, they have associated error bars, which can be determined in different ways. In the case of sharper steps, the usual energy uncertainty (standard deviation) is most useful for effective band determination. For smoother steps, total probability limits (e.g., the 5\% and $95 \%$ limits of a single-band step) can be more useful. In both cases, however, significant overlap of the error bars of two adjacent (effective) bands indicates that alloy disorder has mixed the bands. In semiconductor electronic structure problems, this overlap is most often seen in the valence bands at and near zone-center, where the Heavy- and Light-Hole bands are degenerate in pure materials. In alloys the effective degeneracy typically exists over a small range of $\mathbf{k}$.

\subsection{Interpreting the Unfolded Bands}

As seen above, unfolding disordered (perfect) SC bands to effective (exact) PC bands does not require one to construct the SC Hamiltonian in a PC-periodic basis, but doing so provides additional insights into the expectation values $\bar{E}_{\eta}\left(\mathbf{K}+\mathbf{G}_{m}\right)$ as well as the physics and mathematics of unfolding. Unfolding necessarily presumes a PC-periodic basis, so that the basis 
states are simultaneous eigenstates of the PC-translations, characterized by PC wavevectors $\mathbf{k}_{m}=\mathbf{K}+\mathbf{G}_{m}$. In the case of a disordered SC (i.e., one in which the atomic species and exact positions within the PC vary amongst PCs) one might question the validity of using a PCperiodic basis. So long as it is physically reasonable to use the same number and symmetry of orbitals in each PC, using a PC-periodic basis is justified: The disorder can be confined to the Hamiltonian, not the basis. (If this choice of orbitals is not physically reasonable, then unfolding the SC bands to PC-periodic components is a meaningless exercise. It should also be remembered that a plane-wave basis calculation of the SC eigenstates also uses a PC-periodic basis.) When a PC-periodic basis is used to calculate the SC eigenstates, the disorder (on a PC level) appears as off-diagonal blocks of the Hamiltonian matrix. For example, randomly displaced atoms within the PCs result in randomly varying neighboring-atom Hamiltonian matrix elements: The explicit atomic locations do not appear in the Hamiltonian matrix. In fact, the exact details of how the Hamiltonian matrix elements are calculated are quite immaterial: Two Hamiltonian matrices with identical matrix elements have identical eigenstates. Thus, what matters is that the PC locations are on a periodic lattice, so that in the PC-periodic basis the phase factors are those of a PC-periodic lattice.

One of the advantages in studying the SC Hamiltonian in a PC-periodic basis is that this basis automatically partitions the Hamiltonian matrix elements into two classes: Those that come from the PC-periodic part of the Hamiltonian operator and those that come from the disorder (i.e., non-PC-periodic) part of the Hamiltonian operator. As we show below, the diagonal blocks in the PC-wavevectors, $\mathbf{k}_{m}=\mathbf{K}+\mathbf{G}_{m}$, are due exclusively to the PC-periodic part of the Hamiltonian whereas the off-diagonal blocks $\left(\mathbf{G}_{m}, \mathbf{G}_{n}\right)$ are exclusively due to the disorder part of the Hamiltonian, and account for the alloy scattering. 
To better understand the meaning of the $\bar{E}_{\eta}\left(\mathbf{K}+\mathbf{G}_{m}\right)$, eq. (16), we use the expression for the SC Hamiltonian matrix elements in a PC-periodic basis calculated in Appendix B, eq. (B8). The diagonal $\left(\mathbf{G}_{m}=\mathbf{G}_{n}\right)$ Hamiltonian blocks are of special interest and in certain cases are closely related to the widely-used Virtual Crystal Approximation (VCA) for alloys. From eq. (B8), these are:

$$
\begin{gathered}
\left\langle\omega ; \mathbf{K}^{\prime}+\mathbf{G}_{n}|\hat{H}| \gamma ; \mathbf{K}+\mathbf{G}_{n}\right\rangle=\delta_{\mathbf{K}, \mathbf{K}^{\prime}} \sum_{j=0}^{N_{N N}} e^{i\left(\mathbf{K}+\mathbf{G}_{n}\right) \cdot \mathbf{d}_{j}} \overline{\left\langle\omega ; \mathbf{0}|\hat{H}| \gamma ; \mathbf{d}_{j}\right\rangle} \\
\overline{\left\langle\omega ; \mathbf{0}|\hat{H}| \gamma ; \mathbf{d}_{j}\right\rangle} \square \frac{1}{N_{C}} \sum_{i^{\prime}=1}^{N_{C}}\left\langle\omega ; \boldsymbol{\rho}_{i^{\prime}}|\hat{H}| \gamma ; \boldsymbol{\rho}_{i^{\prime}}+\mathbf{d}_{j}\right\rangle
\end{gathered}
$$

In other words, the diagonal blocks look like those of a PC-periodic Hamiltonian with averaged localized matrix elements. At first glance we might be tempted to conclude that the averaged matrix elements, eq. (18), are simply the familiar VCA, but this is not necessarily true.

To understand the relationship of the averaging in eq. (18) it is helpful to revisit the VCA. It suffices to consider the case of a binary alloy $A_{x} B_{1-x} C$. The onsite $(E)$ and neighboring-atom $(V)$ VCA matrix elements are defined as:

$$
\begin{gathered}
E_{\omega}^{A B C}=x E_{\omega}^{A C}+(1-x) E_{\omega}^{B C}=\frac{1}{N_{C}} \sum_{i^{\prime}=1}^{N_{C}}\left\langle\omega ; \boldsymbol{\rho}_{i^{\prime}}|\hat{H}| \omega ; \boldsymbol{\rho}_{i^{\prime}}\right\rangle \\
V_{\omega, \gamma}^{A B C}=x V_{\omega, \gamma}^{A C}+(1-x) V_{\omega, \gamma}^{B C}
\end{gathered}
$$

From eq. (19) it is obvious that the diagonal matrix elements (same atom, same orbital - we ignore the possibility of same-atom different orbital matrix elements) are averaged exactly as they are in the VCA. For the neighboring-atom matrix elements, the averaging does not necessarily reproduce the VCA: The result of the averaging depends upon whether or not the two pure materials $A C$ and $B C$ have the same lattice constant, because in general neighboring- 
atom matrix elements are functions of the actual distance between the two atoms involved, which we will denote $\mathbf{d}_{j}^{\left(i^{\prime}\right)}$ in reference to eq. (18). In a crystal free of positional disorder, the distance between corresponding neighboring atoms is the same for all PCs, so once again the VCA results. In a system with positional disorder, however, the distances between atoms generally vary randomly and there is generally a distance dependence to the neighboring atom matrix elements (often either Harrison-power-law[20] or exponential). Even the distance between corresponding $A-C$ pairs can vary randomly amongst PCs, for example, so that the averaging in eq. (18) is obviously different from the VCA.

It is important to realize that the linear scaling of the VCA parameters does not imply a zero bowing parameter for semiconductor alloys, since the VCA energies generally have a non-linear dependence on the parameters[21]. The bowing parameter may be described as having contributions from both the VCA and aperiodicity[21]. Second-order perturbation calculations in pseudopotential approaches have been used to calculate the bowing parameter[22], and our own previous work on unfolding AlGaAs random-alloy supercells has produced AlGaAs effective bandgaps with a bowing parameter exceeding that of the VCA alone[4]. The bowing parameter is materials dependent, and after over 60 years of investigation its exact nature is still not fully understood.

Having seen that the diagonal $\left(\mathbf{G}_{m}=\mathbf{G}_{n}\right)$ Hamiltonian blocks are the PC-periodic parts of the Hamiltonian, we expect that the off-diagonal $\left(\mathbf{G}_{m} \neq \mathbf{G}_{n}\right)$ blocks contain only the PC-disorder part of the Hamiltonian. We prove these assertions by partitioning the Hamiltonian into a PCperiodic part $\hat{H}_{P C}$ and a SC-periodic, but PC-aperiodic, or disorder part, $\hat{H}_{D O}$. All of the PCperiodicity is included in $\hat{H}_{P C}$, so that $\hat{H}_{D O}$ has no constant term and thus must average to zero 
over the SC. Any average disorder term is constant, and therefore PC-periodic, and so included in $\hat{H}_{P C}$; were $\hat{H}_{D O}$ to have a non-zero average, we could subtract the average from it and include the average with $\hat{H}_{P C}$. In terms of the localized basis states, then,

$$
\begin{gathered}
\left\langle\omega ; \boldsymbol{\rho}_{i^{\prime}}|\hat{H}| \gamma ; \boldsymbol{\rho}_{i^{\prime}}+\mathbf{d}_{j}\right\rangle=\left\langle\omega ; \mathbf{0}\left|\hat{H}_{P C}\right| \gamma ; \mathbf{d}_{j}\right\rangle+\left\langle\omega ; \boldsymbol{\rho}_{i^{\prime}}\left|\hat{H}_{D O}\right| \gamma ; \boldsymbol{\rho}_{i^{\prime}}+\mathbf{d}_{j}\right\rangle \\
\left\langle\omega ; \boldsymbol{\rho}_{i^{\prime}}\left|\hat{H}_{P C}\right| \gamma ; \boldsymbol{\rho}_{i^{\prime}}+\mathbf{d}_{j}\right\rangle=\left\langle\omega ; \mathbf{0}\left|\hat{H}_{P C}\right| \gamma ; \mathbf{d}_{j}\right\rangle
\end{gathered}
$$

where eq. (22) follows from the PC-periodicity of $\hat{H}_{P C}$.

The partitioned Hamiltonian, eq. (21) is now used to simplify the matrix elements of the SC Hamiltonian in the PC-basis, eq. (B8). Note that the $\hat{H}_{P C}$ matrix element is identical for all PCs. The relevant part is the inner sum over PCs (in curly braces) in eq. (B8):

$$
\begin{aligned}
& \frac{1}{N_{C}} \sum_{i^{\prime}=1}^{N_{C}} e^{i\left(\mathbf{G}_{n}-\mathbf{G}_{m}\right) \cdot \boldsymbol{p}_{i}}\left\langle\omega ; \boldsymbol{\rho}_{i^{\prime}}|\hat{H}| \gamma ; \boldsymbol{\rho}_{i^{\prime}}+\mathbf{d}_{j}\right\rangle= \\
& \left\langle\omega ; \mathbf{0}\left|\hat{H}_{P C}\right| \gamma ; \mathbf{d}_{j}\right\rangle\left[\frac{1}{N_{C}} \sum_{i^{\prime}=1}^{N_{C}} e^{i\left(\mathbf{G}_{n}-\mathbf{G}_{m}\right) \cdot \boldsymbol{p}_{i}}\right]+\frac{1}{N_{C}} \sum_{i^{\prime}=1}^{N_{C}} e^{i\left(\mathbf{G}_{n}-\mathbf{G}_{m}\right) \cdot \boldsymbol{p}_{i}}\left\langle\omega ; \boldsymbol{\rho}_{i^{\prime}}\left|\hat{H}_{D O}\right| \gamma ; \boldsymbol{\rho}_{i^{\prime}}+\mathbf{d}_{j}\right\rangle
\end{aligned}
$$

The Fourier sum in square brackets above simplifies to $\delta_{m, n}[23]$. Thus

$$
\frac{1}{N_{C}} \sum_{i^{\prime}=1}^{N_{C}} e^{i\left(\mathbf{G}_{n}-\mathbf{G}_{m}\right) \cdot \boldsymbol{p}_{i}}\left\langle\omega ; \boldsymbol{\rho}_{i^{\prime}}|\hat{H}| \gamma ; \boldsymbol{\rho}_{i^{\prime}}+\mathbf{d}_{j}\right\rangle=\left\langle\omega ; \mathbf{0}\left|\hat{H}_{P C}\right| \gamma ; \mathbf{d}_{j}\right\rangle \delta_{m, n}+\frac{1}{N_{C}} \sum_{i^{\prime}=1}^{N_{C}} e^{i\left(\mathbf{G}_{n}-\mathbf{G}_{m}\right) \cdot \boldsymbol{p}_{i}}\left\langle\omega ; \boldsymbol{\rho}_{i^{\prime}}\left|\hat{H}_{D O}\right| \gamma ; \boldsymbol{\rho}_{i^{\prime}}+\mathbf{d}_{j}\right\rangle
$$

We complete the proof by using eq. (24) in eq. (B8) to calculate the off-diagonal and diagonal blocks. First, we simplify the off-diagonal Hamiltonian blocks,

$$
\left\langle\omega ; \mathbf{K}^{\prime}+\mathbf{G}_{m}|\hat{H}| \gamma ; \mathbf{K}+\mathbf{G}_{n}\right\rangle=\delta_{\mathbf{K}, \mathbf{K}} \sum_{j=0}^{N_{N}} e^{i\left(\mathbf{K}+\mathbf{G}_{n}\right) \cdot \mathbf{d}_{j}}\left\{\frac{1}{N_{C}} \sum_{i^{\prime}=1}^{N_{C}} e^{i\left(\mathbf{G}_{n}-\mathbf{G}_{m}\right) \cdot \mathbf{P}_{i}}\left\langle\omega ; \boldsymbol{\rho}_{i^{\prime}}\left|\hat{H}_{D O}\right| \gamma ; \boldsymbol{\rho}_{i^{\prime}}+\mathbf{d}_{j}\right\rangle\right\}, m \neq n
$$


Note that the sum over PCs automatically eliminates all PC-periodic components from the offdiagonal matrix element, eq. (25). Therefore, the partitioning into PC-periodic and disorder terms is automatic, and the off-diagonal blocks contain exclusively the PC-disorder part of the SC Hamiltonian without any PC-periodic component. Next we simplify the diagonal blocks,

$$
\left\langle\omega ; \mathbf{K}^{\prime}+\mathbf{G}_{n}|\hat{H}| \gamma ; \mathbf{K}+\mathbf{G}_{n}\right\rangle=\delta_{\mathbf{K}, \mathbf{K}^{\prime}} \sum_{j=0}^{N_{N}} e^{i\left(\mathbf{K}+\mathbf{G}_{n}\right) \cdot \mathbf{d}_{j}}\left\{\left\langle\omega ; \mathbf{0}\left|\hat{H}_{P C}\right| \gamma ; \mathbf{d}_{j}\right\rangle+\frac{1}{N_{C}} \sum_{i^{\prime}=1}^{N_{C}}\left\langle\omega ; \boldsymbol{\rho}_{i^{\prime}}\left|\hat{H}_{D O}\right| \gamma ; \boldsymbol{\rho}_{i^{\prime}}+\mathbf{d}_{j}\right\rangle\right\}
$$

However by our partitioning, the disorder term averages to zero over the SC and the diagonal blocks are thus the purely PC-periodic parts:

$$
\left\langle\omega ; \mathbf{K}^{\prime}+\mathbf{G}_{n}|\hat{H}| \gamma ; \mathbf{K}+\mathbf{G}_{n}\right\rangle=\delta_{\mathbf{K}, \mathbf{K}} \sum_{j=0}^{N_{N}} e^{i\left(\mathbf{K}+\mathbf{G}_{n}\right) \cdot \mathbf{d}_{j}}\left\langle\omega ; \mathbf{0}\left|\hat{H}_{P C}\right| \gamma ; \mathbf{d}_{j}\right\rangle
$$

where we have from eq. (18),

$$
\overline{\left\langle\omega ; \mathbf{0}|\hat{H}| \gamma ; \mathbf{d}_{j}\right\rangle}=\left\langle\omega ; \mathbf{0}\left|\hat{H}_{P C}\right| \gamma ; \mathbf{d}_{j}\right\rangle=\frac{1}{N_{C}} \sum_{i^{\prime}=1}^{N_{C}}\left\langle\omega ; \boldsymbol{\rho}_{i^{\prime}}|\hat{H}| \gamma ; \boldsymbol{\rho}_{i^{\prime}}+\mathbf{d}_{j}\right\rangle
$$

We remark that the average, eq. (18), necessarily encompasses all of the PC-periodic content ot the Hamiltonian matrix elements. Therefore separating the Hamiltonian into PC-periodic and disorder terms in a PC-periodic basis shows that the diagonal $\left(\mathbf{G}_{m}=\mathbf{G}_{n}\right)$ blocks contain all of the PC-periodicity and the off-diagonal $\left(\mathbf{G}_{m} \neq \mathbf{G}_{n}\right)$ blocks all of the disorder.

We now turn to the relationship of the expectation values, $\bar{E}_{\eta}\left(\mathbf{k}_{m}\right), \mathbf{k}_{m}=\mathbf{K}+\mathbf{G}_{m}$, eq. (16) to the diagonal Hamiltonian blocks in the PC-periodic basis. The PC band states, eq. (2) are clearly expansions in the PC-periodic basis states, eq. (B1), so we might be tempted to conclude that the $\bar{E}_{\eta}\left(\mathbf{k}_{m}\right)$ are eigenvalues of the Hamiltonian diagonal $\left(\mathbf{k}_{m}, \mathbf{k}_{m}\right)$ block, denoted $\underline{\mathbf{H}}\left(\mathbf{k}_{m}, \mathbf{k}_{m}\right)$. 
(Note that this block matrix is Hermitian.) However, this conclusion is generally incorrect, even in the case of a strain-free alloy. The $\bar{E}_{\eta}\left(\mathbf{k}_{m}\right)$ are expectation values of states with respect to the $\underline{\mathbf{H}}\left(\mathbf{k}_{m}, \mathbf{k}_{m}\right)$ block, but they are not necessarily eigenstates. In linear algebra and matrix theory they are members of the field of values of the matrix $\underline{\mathbf{H}}\left(\mathbf{k}_{m}, \mathbf{k}_{m}\right)$, and they satisfy $\varepsilon_{\min }\left(\mathbf{k}_{m}\right) \leq \bar{E}_{\eta}\left(\mathbf{k}_{m}\right) \leq \varepsilon_{\max }\left(\mathbf{k}_{m}\right)$, where $\varepsilon_{\min }\left(\mathbf{k}_{m}\right)$ and $\varepsilon_{\max }\left(\mathbf{k}_{m}\right)$ respectively denote the minimum and maximum eigenvalues of the diagonal block matrix $\underline{\mathbf{H}}\left(\mathbf{k}_{m}, \mathbf{k}_{m}\right)$ [24].

Because only the diagonal elements of the block matrix $\underline{\mathbf{H}}\left(\mathbf{k}_{m}, \mathbf{k}_{m}\right)$ in the PC-band state basis, eq. (2) are available, it does not seem that the $\left|\psi_{\eta}\left(\mathbf{k}_{m}\right)\right\rangle$ can be exactly determined, even as expansions in the eigenstates of the block $\underline{\mathbf{H}}\left(\mathbf{k}_{m}, \mathbf{k}_{m}\right)$. We might therefore use as a starting point for approximate "alloy eigenstates" the eigenvectors of the diagonal Hamiltonian block $\underline{\mathbf{H}}\left(\mathbf{k}_{m}, \mathbf{k}_{m}\right)$, but in cases where $\bar{E}_{\eta}\left(\mathbf{k}_{m}\right)$ is not close to one of the eigenvalues $\varepsilon_{n}\left(\mathbf{k}_{m}\right)$ the approximation will not be good. Even taking orthonormal linear combinations of several diagonal block eigenstates to fit several of the $\bar{E}_{\eta}\left(\mathbf{k}_{m}\right)$ does not guarantee that the trial states are the correct ones. As usual in quantum mechanics, approximate eigenvalues are much easier to obtain than are approximate eigenstates.

\section{Results}

We demonstrate the use of SC-band unfolding to find the effective PC-bands for a simple one-dimensional model. Each PC has two atoms, with a single $s$-orbital on the cation and a single $p_{z}$-orbital on the anion; only the onsite and nearest-neighbor Hamiltonian matrix elements are included. This model is a simplified version of the second-near-neighbor diatomic model for 
III-V materials introduced by Sai-Halasz, Esaki, and Harrison[25] and previously used by one of us[26] in tunneling calculations. Here we consider a random-alloy chain consisting of cells of two types $A C$ and $B C$. Although the anion $(C)$ is common, a valence-band offset is built in, $\varepsilon_{p}^{A C} \neq \varepsilon_{p}^{B C}$, as one finds in semiconductor interfaces. The interface is always taken at the anion layer, and when an anion has an $A$-cation on one side and a $B$-cation on the other, its onsite energy is taken as $\left(\varepsilon_{p}^{A C}+\varepsilon_{p}^{B C}\right) / 2$. The nearest-neighbor parameters are the same as in bulk: with the $A$-cation the parameter is $\pm V_{s p}^{A C}$ and with the $B$-cation it is $\mp V_{s p}^{B C}$; the sign change is due to the fact that one cation is nearest the negative lobe (+ sign, as an attractive potential is assumed) while the other is nearest the positive lobe (- sign). The parameter values used here are listed in Table I.

Figure 2 shows the projection and cumulative probabilities at $k=0$ for a random alloy chain $A_{0.5} B_{0.5} C$; the chain consists of 1000 PCs. The dots are the projection probabilities and the solid line is the cumulative probability. With the supercell energies sorted in ascending order, the cumulative probability for energy $E_{M}, 1 \leq M \leq N_{O} N_{C}$ at PC wavevector $\mathbf{k}_{n}=\mathbf{K}+\mathbf{G}_{n}$ is defined as the sum of the projection probabilities for SC eigenstates of energies $E_{p} \leq E_{M}$ [4]:

$$
\mathcal{P}_{n, \text { cum }}\left(E_{M}\right)=\sum_{p=1}^{M} \mathcal{P}_{p, n}
$$

The cumulative probability step up to the valence band is rough, and while the initial jump for the conduction band is sharp, the final ascent to cumulative probability 2.0 is slow. Partly this is due to the worst-case nature of the 50/50 alloy and partly it is the fact that the valance band is the lowest band in the material while the conduction band is the highest. The lowest band starts with the projection probabilities at the lowest energies, while the highest band ends with the projection probabilities at the highest energies. 
A useful metric for defining effective bands is the band probability, which is based on the cumulative probability. For a non-degenerate band, the band probability for the $n$-th band (as a function of energy) is defined as $(n-1)$. In the two-band model here, this means that when the cumulative probability has reached 1.0 the first (valence) band has been passed, and when it reaches 2.0 the second (conduction) band has been passed. Because these bands are effective (approximate) they have energy uncertainties; one useful way to quantify these is via band probability brackets. For example, the $(25 \%, 75 \%)$ band probability bracket corresponds to the cumulative probability range $0.25 \leq \mathscr{P}_{\text {cum }} \leq 0.75$ for the valence $(n=1)$ band and $1.25 \leq \mathcal{P}_{\text {cum }} \leq 1.75$ for the conduction $(n=2)$ band

Figure 3 shows the bands for the random chain alloy $A_{0.5} B_{0.5} C$ of Fig. 2 . The blue lines show the bulk dispersions for the two pure materials. The $A C$ bands are the higher conduction band and lower valence band while the $B C$ bands are the lower conduction band and higher valence band. The red lines show the effective (average) energies for the alloy chain and the bars give energy brackets. The grey bars bracket the $(5 \%, 95 \%)$ band probability points while the black bars bracket the $(25 \%, 75 \%)$ points. First, note that the effective bands (red) fall near the midpoint of those of the two constituent materials. While this behavior is in-line with the VCA, note as well the significant energy spreads, which cannot be determined when using the VCA, as the VCA energies are eigenvalues of the VCA Hamiltonian. As shown above, the band energies $\bar{E}_{\eta}\left(\mathbf{k}_{m}\right)$ are only expectation values with respect to the diagonal PC-basis Hamiltonian blocks, and therefore are not eigenvalues of the disordered SC Hamiltonian. Their finite energy spread is due to the disorder in the $\mathrm{SC}$, which imparts to them finite, alloy-scattering lifetimes.

Considering the energy brackets at $k=0$, we see that they follow directly from the projection probabilities and cumulative probabilities in Fig. 2. In the conduction band note that the 0.25 
band probability point (cumulative probability 1.25 ) occurs at around $0.3 \mathrm{eV}$; in the valence band the corresponding point occurs at around $-0.5 \mathrm{eV}$. Both features are clearly evident in the effective bandstructure, Fig. 3. Finally, note in Fig. 2 that significant projection probabilities occur up to nearly $1.0 \mathrm{eV}$, and this behavior is evident in Fig. 3, where the upper grey bar (95\% band probability) terminates at just under $1.0 \mathrm{eV}$. This extended topping-out is what one should expect for the highest band in a model.

\section{Conclusions}

We have shown that Brillouin-zone unfolding applied to a random-alloy SC can give the effective (i.e., approximate) PC-bandstructure for the random-alloy, which can be measured. Our development emphasizes the generality of the procedure by formulating it as a discrete Fourier transform. Projecting the SC eigenstates onto a PC-periodic basis leads to the effective band energies. We have shown that these effective bands are expectation values with respect to the diagonal Hamiltonian blocks in a PC-periodic basis, although not necessarily eigenvalues of these blocks. In a disordered SC, the effective bands have finite energy spreads corresponding to finite alloy-scattering lifetime: The effective bands are only expectation values, not eigenvalues. Thus it is not necessary to describe the unfolding and approximate band calculation in terms of elaborate mathematics: Discrete Fourier methods like those widely employed in audio and video signal processing are fully sufficient.

\section{Acknowledgements}

This work was supported in part by the Center for Low Energy Systems Technology (LEAST), one of six centers of STARnet, a Semiconductor Research Corporation program sponsored by 
MARCO and DARPA. The use of nanoHUB.org computational resources operated by the Network for Computational Nanotechnology funded by the US National Science Foundation under grant EEC-1227110, EEC-0228390, EEC-0634750, OCI-0438246, and OCI-0721680 is gratefully acknowledged. This work was also supported in part by the National Science Foundation under Grant Number 1125017. 


\section{Appendix A: Existence of a discrete real-space grid}

It is well known that boundary conditions and a finite real-space grid force $k$-space quantization. That is, the $\mathbf{k}$ values also conform to a finite grid. Examples include, in three dimensions, applying periodic boundary conditions to a large number of primitive cells; and in one dimension, the discretized effective-mass Schrödinger equation[27]. Here we show that the converse is true: A finite grid of $k$ values automatically implies a finite real-space grid.

We demonstrate this property of a finite $k$-space grid in one dimension, and since our interest is in periodic functions we treat that case. The starting point is the Fourier series representation for a continuous, periodic, well-behaved function, $f(x)$, of period $X_{0}, f\left(x+X_{0}\right)=f(x)$ :

$$
f(x)=\sum_{n=-\infty}^{\infty} c_{n} \exp \left(i n k_{0} x\right), \quad c_{n}=\frac{1}{X_{0}} \int_{X_{0}} f(x) \exp \left(-i n k_{0} x\right) d x, \quad k_{0}=\frac{2 \pi}{X_{0}}
$$

Now, we investigate what happens to the Fourier series representation when take only a finite number of harmonics, $N$, just as we would do in any numerical calculation (e.g., of a semiconductor bandstructure with a plane-wave basis). The first step in the truncation process is to divide the sum in eq. (A1) into terms to be retained and those to be discarded:

$$
f(x)=c_{0}+\sum_{n=1}^{N-1}\left[c_{n} \exp \left(i n k_{0} x\right)+c_{-n} \exp \left(-i n k_{0} x\right)\right]+\sum_{n=N}^{\infty}\left[c_{n} \exp \left(i n k_{0} x\right)+c_{-n} \exp \left(-i n k_{0} x\right)\right]
$$

In eq. (A2) at this point there are $2 N-1$ harmonics between the DC term and the first sum; we will later reduce this to only $N$. We want to discard the last sum, but before doing so, we rewrite it as a double sum, changing variables to:

$$
\begin{gathered}
n=p N+m, \quad p=1,2, \ldots, \infty ; \quad m=0,1, \ldots,(N-1) \\
f(x)=c_{0}+\sum_{m=1}^{N-1}\left[c_{m} \exp \left(i m k_{0} x\right)+c_{-m} \exp \left(-i m k_{0} x\right)\right]+\sum_{p=1}^{\infty}\left[c_{p N} \exp \left(i p N k_{0} x\right)+c_{-p N} \exp \left(-i p N k_{0} x\right)\right]
\end{gathered}
$$




$$
+\sum_{m=1}^{N-1} \sum_{p=1}^{\infty}\left\{c_{p N+m} \exp \left[i(p N+m) k_{0} x\right]+c_{-(p N+m)} \exp \left[-i(p N+m) k_{0} x\right]\right\}
$$

In eq. (A4) we have separated out the $m=0$ terms into the second sum.

Clearly, if we truncate the series by retaining only the DC term and first sum, we will no longer exactly represent the function at arbitrary points, $x$. However, it turns out that if we evaluate the function only at certain discrete points we can exactly represent the function with only $N$ harmonics. These points, which define a real-space grid, are given by:

$$
x_{n}=\frac{n}{N} X_{0}, \quad n=0,1, \ldots,(N-1)
$$

First, observe that higher harmonics now vanish from the sum since,

$$
\exp \left[ \pm i p N k_{0} x_{n}\right]=\exp \left[ \pm i p N \frac{2 \pi}{X_{0}} \frac{n}{N} X_{0}\right]=\exp ( \pm i 2 \pi p n)=1
$$

Next, observe that the remaining negative low-order harmonics likewise disappear. For the negative harmonics in eq. (A4) we change variables on the low-order sum to:

$$
l=N-m, \quad l=1, \ldots,(N-1) \Leftrightarrow m=(N-1), \ldots, 1
$$

Thus, using eq. (A6),

$$
\begin{gathered}
\sum_{m=1}^{N-1} c_{-m} \exp \left(-i m k_{0} x_{n}\right)=\sum_{l=1}^{N-1} c_{l-N} \exp \left[-i(N-l) k_{0} x_{n}\right]=\sum_{l=1}^{N-1} c_{l-N} \exp \left[i l k_{0} x_{n}\right] \\
\sum_{m=1}^{N-1} \sum_{p=1}^{\infty}\left\{c_{-(p N+m)} \exp \left[-i(p N+m) k_{0} x_{n}\right]\right\}=\sum_{l=1}^{N-1} \sum_{p=1}^{\infty}\left\{c_{-(p N+N-l)} \exp \left[-i(p N+N-l) k_{0} x_{n}\right]\right\}= \\
\sum_{l=1}^{N-1} \sum_{p=1}^{\infty}\left[c_{-(p+1) N+l} \exp \left(i l k_{0} x_{n}\right)\right]
\end{gathered}
$$


The negative harmonic $(l-N) k_{0}$ has the same value as the positive harmonic $l k_{0}$ when evaluated at one of the $N$ discrete points. Substituting eqs. (A6), (A8), and (A9) into eq. (A4) we see that the function is indeed exactly represented by $N$ harmonics:

$$
\begin{gathered}
f\left(x_{n}\right)=d_{0}+\sum_{m=1}^{N-1} d_{m} \exp \left(i m k_{0} x_{n}\right) \\
d_{0}=c_{0}+\sum_{p=1}^{\infty}\left[c_{p N}+c_{-p N}\right], \quad d_{m}=\left[c_{m}+c_{m-N}\right]+\sum_{p=1}^{\infty}\left[c_{p N+m}+c_{-(p+1) N+m}\right]
\end{gathered}
$$

Eq. (A10) is called the discrete Fourier series representation of the function[17]. We remark that this result is intuitively satisfying since a real space basis for the function values is the set of $N$ Kronecker delta functions $\left\{\delta_{n, l}\right\}, l, n=0,1, \ldots,(N-1)$ giving the same number of basis functions in both real and $k$-space. We can thus exactly represent $N$ samples of the function with only $N$ harmonics, and the function values are exact only on the discrete grid points. Moreover, specifying the coefficients, $d_{m}$, in the discrete Fourier transform automatically fixes the values of $f$ on the real-space grid points, $x_{n}$.

\section{Appendix B: SC Hamiltonian matrix elements in a PC-periodic basis}

Here we calculate the matrix elements of the SC Hamiltonian in a PC-periodic basis; these are used in Sec. II.C. above. The solid consists of $N_{S}$ identical SCs, each of which has $N_{C}$ PCs. We use the same basis functions in each PC even though the precise atom positions or species may differ from PC to PC. The PC-periodic basis for this solid is defined as:

$$
\left|\omega ; \mathbf{K}+\mathbf{G}_{m}\right\rangle=\frac{1}{\sqrt{N_{S} N_{C}}} \sum_{j=1}^{N_{S}} \sum_{i=1}^{N_{C}} e^{i \mathbf{K} \cdot \mathbf{R}_{j}} e^{i\left(\mathbf{K}+\mathbf{G}_{m}\right) \cdot \mathbf{p}_{i}}\left|\omega ; \mathbf{R}_{j}+\mathbf{\rho}_{i}\right\rangle
$$


Note that the states (B1) need not be band basis states. It is most convenient to use a basis of Bloch sums of a single orbital type. In eq. (B1), $\mathbf{R}_{j}$ is the location of the $j$-th $\mathrm{SC}, \boldsymbol{\rho}_{i}$ is the location of the $i$-th PC, relative to its SC origin, and $\omega$ is the orbital type. Eq. (B1) has been simplified by observing that $\mathbf{G}_{m} \mathbf{R}_{j}=2 \pi \times$ integer. The Hamiltonian matrix elements in this basis are:

$$
\begin{aligned}
& \left\langle\omega ; \mathbf{K}^{\prime}+\mathbf{G}_{m}|\hat{H}| \gamma ; \mathbf{K}+\mathbf{G}_{n}\right\rangle= \\
& \frac{1}{N_{S} N_{C}} \sum_{j, j^{\prime}=1}^{N_{S}} \sum_{i, i^{\prime}=1}^{N_{C}}\left\{e^{i \mathbf{K} \cdot \mathbf{R}_{j}} e^{-i \mathbf{K}^{\prime} \cdot \mathbf{R}_{j^{\prime}}} e^{-i\left(\mathbf{K}^{\prime}+\mathbf{G}_{m}\right) \cdot \boldsymbol{p}^{\prime}} e^{i\left(\mathbf{K}+\mathbf{G}_{n}\right) \cdot \boldsymbol{\rho}_{i}}\left\langle\omega ; \mathbf{R}_{j^{\prime}}+\boldsymbol{\rho}_{i^{\prime}}|\hat{H}| \gamma ; \mathbf{R}_{j}+\boldsymbol{\rho}_{i}\right\rangle\right\}
\end{aligned}
$$

Because the Hamiltonian is SC-periodic we shift the SC locations,

$$
\begin{gathered}
\mathbf{R}_{j}=\mathbf{R}_{l}+\mathbf{R}_{j^{\prime}} \\
\left\langle\omega ; \mathbf{R}_{j^{\prime}}+\boldsymbol{\rho}_{i^{\prime}}|\hat{H}| \gamma ; \mathbf{R}_{j}+\boldsymbol{\rho}_{i}\right\rangle=\left\langle\omega ; \mathbf{R}_{j^{\prime}}+\boldsymbol{\rho}_{i^{\prime}}|\hat{H}| \gamma ; \mathbf{R}_{j^{\prime}}+\mathbf{R}_{l}+\boldsymbol{\rho}_{i}\right\rangle=\left\langle\omega ; \boldsymbol{\rho}_{i^{\prime}}|\hat{H}| \gamma ; \mathbf{R}_{l}+\boldsymbol{\rho}_{i}\right\rangle
\end{gathered}
$$

and now sum over $l$ instead of $j$. As the matrix elements are now independent of $j^{\prime}$ that sum becomes an overall factor multiplying the remaining sums $l, i, i^{\prime}$, and using the Fourier sum[23],

$$
\frac{1}{N_{S}} \sum_{j^{\prime}=1}^{N_{S}} e^{i\left(\mathbf{K}-\mathbf{K}^{\prime}\right) \cdot \mathbf{R}_{j^{\prime}}}=\delta_{\mathbf{K}, \mathbf{K}^{\prime}}
$$

to find,

$$
\left\langle\omega ; \mathbf{K}^{\prime}+\mathbf{G}_{m}|\hat{H}| \gamma ; \mathbf{K}+\mathbf{G}_{n}\right\rangle=\frac{\delta_{\mathbf{K}, \mathbf{K}^{\prime}}}{N_{C}} \sum_{l=1}^{N_{S}} \sum_{i, i^{\prime}=1}^{N_{C}}\left\{e^{i \mathbf{K} \cdot \mathbf{R}_{l}} e^{i \mathbf{K} \cdot\left(\boldsymbol{\rho}_{i}-\boldsymbol{\rho}_{i^{\prime}}\right)} e^{-i \mathbf{G}_{m} \cdot \boldsymbol{\rho}_{i}^{\prime}} e^{i \mathbf{G}_{n} \cdot \boldsymbol{\rho}_{i}}\left\langle\omega ; \boldsymbol{\rho}_{i^{\prime}}|\hat{H}| \gamma ; \mathbf{R}_{l}+\boldsymbol{\rho}_{i}\right\rangle\right\}
$$

Because the basis is PC-periodic, the PC locations are ideal, even though the Hamiltonian itself is not PC-periodic. Displacements of the atoms within the PCs do not appear explicitly. Instead, they alter the neighboring-atom Hamiltonian matrix elements. That means that for a given PC at $\boldsymbol{\rho}_{i^{\prime}}$, the number of neighbors and distances to them is the same, independent of the specific PC. 
Thus the sums over SCs and PCs may be collapsed into a single sum over neighbors (in principle all neighbors, not just the nearest). Thus,

$$
\mathbf{R}_{l}+\boldsymbol{\rho}_{i}=\boldsymbol{\rho}_{i^{\prime}}+\mathbf{d}_{j}, j=0,1, \ldots, N_{N} ; \mathbf{d}_{0}=\mathbf{0}
$$

where $N_{N} \leq N_{C} N_{S}-1$ is the number of neighbors, with $j=0$ designating the same atom. Making this change in eq. (B6), we find for the matrix elements in the PC-periodic basis,

$$
\left\langle\omega ; \mathbf{K}^{\prime}+\mathbf{G}_{m}|\hat{H}| \gamma ; \mathbf{K}+\mathbf{G}_{n}\right\rangle=\delta_{\mathbf{K}, \mathbf{K}^{\prime}} \sum_{j=0}^{N_{N}} e^{i\left(\mathbf{K}+\mathbf{G}_{n}\right) \cdot \mathbf{d}_{j}}\left\{\frac{1}{N_{C}} \sum_{i^{\prime}=1}^{N_{C}} e^{i\left(\mathbf{G}_{n}-\mathbf{G}_{m}\right) \cdot \boldsymbol{\cdot}_{i}}\left\langle\omega ; \boldsymbol{\rho}_{i^{\prime}}|\hat{H}| \gamma ; \boldsymbol{\rho}_{i^{\prime}}+\mathbf{d}_{j}\right\rangle\right\}
$$

Note that we have changed the order of summation, summing over neighbors last. A few observations regarding eq. (B8) are helpful. First, note that were the Hamiltonian PC-periodic, we could use the same process as in eq. (B5) to find that the matrix elements would be diagonal in $\mathbf{G}_{m}$ as well. Second, note that for a given neighboring atom, the sum in curly braces is a phase-weighted average matrix element over PCs in the SC. 


\section{References}

[1] J. Hwang, C. K. Shih, P. Pianetta, G. D. Kubiak, R. H. Stulen, L. R. Dawson, Y.-C. Pao, and J. S. Harris, Jr., Appl. Phys. Lett. 52 (1988) 308.

[2] J. Endicott, A. Patane, D. Maude, L. Eaves, M. Hopkinson, and G. Hill, Phys. Rev. B 72 (2005) 041306.

[3] T. B. Boykin and G. Klimeck, Phys. Rev. B. 71 (2005) 115215.

[4] T. B. Boykin, N. Kharche, G. Klimeck, and M. Korkusinski, J. Phys.: Condens. Matt. 19 (2007) 036203.

[5] T. B. Boykin, N. Kharche, and G. Klimeck, Phys. Rev. B 76 (2007) 035310.

[6] T. B. Boykin, A. Ajoy, H. Ilatikhameneh, M. Povolotskyi, and G. Klimeck, Phys. Rev. B 90 (2014) 205214.

[7] V. Popescu and A. Zunger Phys. Rev. Lett. 104 (2010) 236403.

[8] V. Popescu and A. Zunger, Phys. Rev. B 85 (2012) 085201.

[9] A. Ajoy, K. V. R. M. Murali, and S. Karmalkar, J. Phys.: Condens. Matt. 24 (2012) 055504. 
[10] P. B. Allen, T. Berlijn, D. A. Casavant, and J. M. Soler, Phys. Rev. B 87 (2013) 085322; Erratum: Phys. Rev. B 87 (2013) 239904(E).

[11] W. Ku, T. Berlijn, and C.-C. Lee, Phys. Rev. Lett. 104 (2010) 216401.

[12] T. Berlijn, Phys. Rev. B 89 (2014) 104511.

[13] C.-C. Lee, Y. Yamada-Takamura, and T. Ozaki, J. Phys.: Condens. Matt. 25 (2013) 345501.

[14] H. Huang, F. Zheng, P. Zheng, J. Wu, B.-L. Gu, and W. Duan, New. J. Phys. 16 (2014) 033304.

[15] P. V. C. Medeiros, S. Stafström, and J. Björk, Phys. Rev. B 89 (2014) 041407(R).

[16] O. E. Peil, A. V. Ruban, and B. Johansson, Phys. Rev. B 85 (2012) 165140.

[17] B. P. Lathi, Signal Processing and Linear Systems, Oxford University Press, New York, 2000, Ch. 10.

[18] T. B. Boykin, N. Kharche, and G. Klimeck, Eur. J. Phys. 27 (2006) 5.

[19] T. B. Boykin, N. Kharche, and G. Klimeck, Physica E 41 (2009) 490. 
[20] Walter A. Harrison, Elementary Electronic Structure, World Scientific, New Jersey, 1999, Sec. 5.3.C.

[21] J. A. Van Vechten and T. K. Bergstresser, Phys. Rev. B 1 (1970) 3351.

[22] M. Morifuji and F. Ishikawa, Physica B 485 (2016) 89.

[23] See, for example, N. W. Ashcroft and N. D. Mermin, Solid State Physics, Saunders, Philadelphia, 1976, Appendix F.

[24] P. Lancaster and M. Tismenetsky, The Theory of Matrices, 2ed. With Applications, New York, Academic Press, 1985, Sec. 8.1.

[25] G. A. Sai-Halasz, L. Esaki, and W. A. Harrison, Phys. Rev. B 18 (1978) 2812.

[26] T. B. Boykin, Phys. Rev. B 54 (1996) 7670.

[27] T. B. Boykin and G. Klimeck, Eur. J. Phys. 25 (2004) 503. 
Table I: Parameters for the one-dimensional chain; all values are in $\mathrm{eV}$.

\begin{tabular}{|l|l|l|l|}
\hline & $\varepsilon_{s}$ & $\varepsilon_{p}$ & $V_{s p}$ \\
\hline$A C$ & 0.5 & -0.6 & 0.5 \\
\hline$B C$ & 0.3 & -0.2 & 0.3 \\
\hline
\end{tabular}




\section{Figure Captions}

Figure 1: Translation vectors and Brillouin zones for a $2 \times 2 \mathrm{PC}$ supercell in a square lattice.

(a) Direct lattice vectors for the SC $\left(\mathbf{R}_{j}\right)$ and PC $\left(\boldsymbol{\rho}_{l}\right)$; each SC has 4 PCs. (b) Reciprocal lattices and Brillouin zones for the SC and PC of (a). The four PC wavevectors $\mathbf{k}_{n}=\mathbf{K}+\mathbf{G}_{n}, n=1, \ldots, 4$, are associated with the single SC wavevector $\mathbf{K}$.

Figure 2: Cumulative (red solid line) and projection (blue dots) probabilities at $k=0$ for a random alloy chain $A_{0.5} B_{0.5} C$; the chain consists of 1000 PCs (color online only). When the cumulative probability has reached 1.0 the first (valence) band has been passed, and when it reaches 2.0 the second (conduction) band has been passed. The band probability for the $n$-th band (as a function of energy) is defined as $(n-1)$. For example, the $(5 \%, 95 \%)$ band probability bracket corresponds to the cumulative probability range $0.05 \leq \mathscr{P}_{\text {cum }} \leq 0.95$ for the valence $(n=1)$ band and $1.05 \leq \mathcal{P}_{\text {cum }} \leq 1.95$ for the conduction $(n=2)$ band.

Figure 3: Effective bands for a random alloy chain $A_{0.5} B_{0.5} C$ of Fig. 2 (see text). The blue lines show the bulk dispersions for the two pure materials: Solid lines ( $A C$ bands) or dashed lines $(B C$ bands), color online only. The red lines show the effective (average) energies for the alloy chain and the bars give energy brackets. The grey bars bracket the $(5 \%, 95 \%)$ band probability points while the black bars bracket the $(25 \%, 75 \%)$ points. The inset at top shows a section of the chain. 


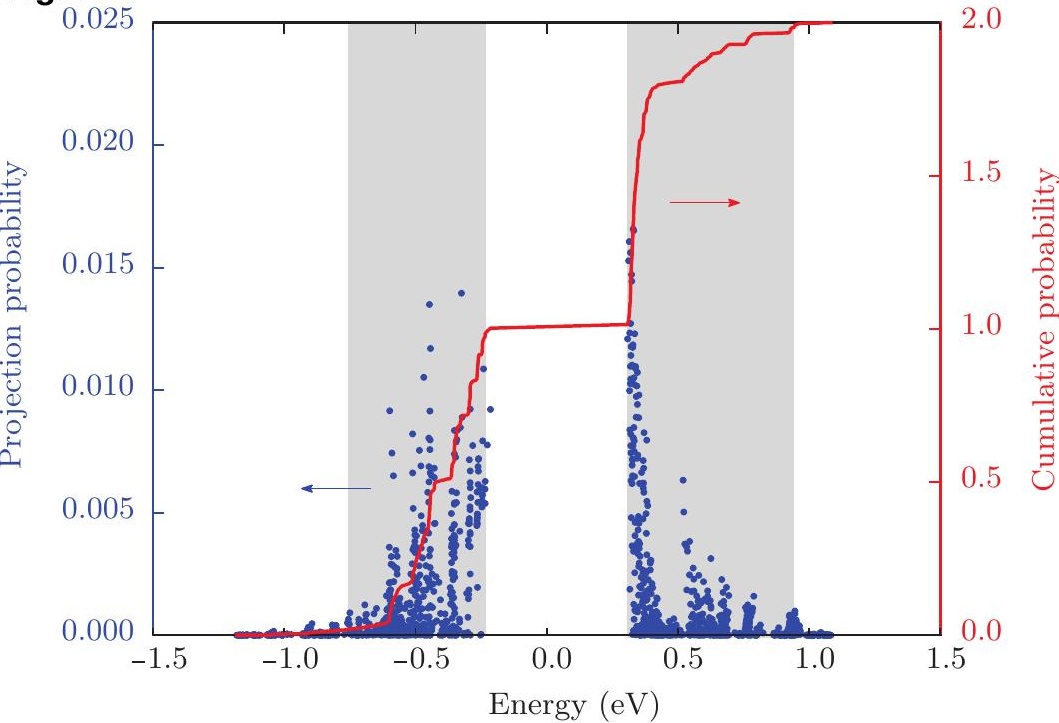


1.5

Figure3

8

1.0
0.5
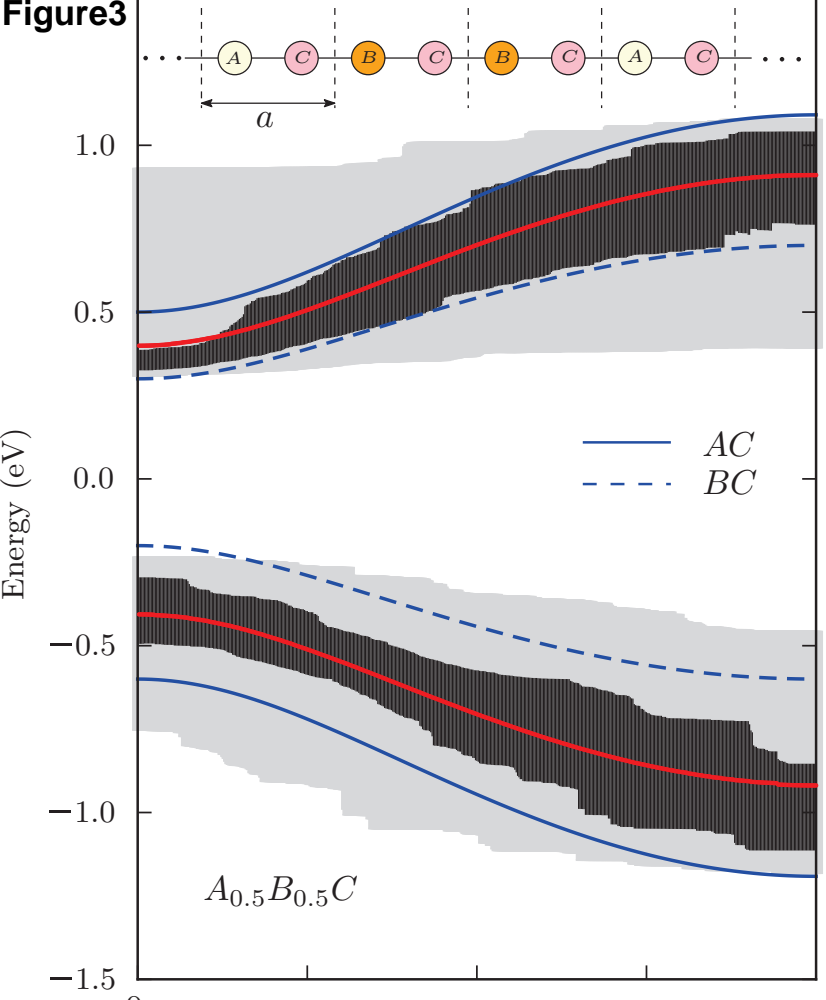

0 